\title{
Hommages : Annette Duchêne et Georges Marblé
}

\section{Annette Duchêne}

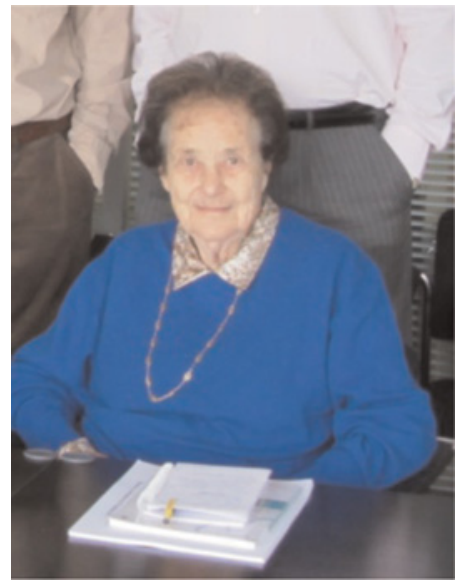

Nous avons appris avec tristesse la disparition de notre collègue Annette le 13 janvier dernier. Venant du domaine des rayonnements ionisants, elle a assisté à l'émergence de la question des rayonnements non ionisants (RNI). Elle ne s'est pas contentée d'en être spectatrice, agissant au cœur de la principale organisation du domaine pendant de nombreuses années, l'ICNIRP.

Annette était spécialisée en réglementation de la protection contre les rayonnements ionisants au CEA lorsque, devant la montée des sollicitations concernant les RNI, elle a été chargée de mission pour traiter des problèmes posés pour la protection des personnes exposées. Dès la constitution du groupe de travail de l'International Radiation Protection Association (IRPA) sur les RNI en 1974, Henri Jammet lui a demandé d'en assurer le secrétariat scientifique. Le groupe de travail a conclu ses travaux par des recommandations en faveur de la création d'une autorité internationale compétente spécifique pour les RNI. Le Conseil d'administration de l'IRPA ayant jugé cette création prématurée, un autre groupe de travail a pris le relais, toujours avec Annette comme secrétaire. Enfin, lors de la création par l'IRPA du Comité international sur les rayonnements non ionisants (INIRC) en 1977, elle en a été officiellement nommée secrétaire scientifique, travaillant successivement avec Henri Jammet, président de 1977 à 1988, puis Mike Repacholi qui lui a succédé.

Polyglotte et ayant des relations avec de nombreux laboratoires, Annette a facilité l'organisation de réunions de travail, de colloques et la rédaction de plusieurs recommandations, sans parler des nombreux autres documents préparés par ou avec le Comité (Environmental Health Criteria de l'OMS ou Occupational Safety and Health Series de l'OIT par exemple). Ceci a permis à l'INIRC d'être rapidement reconnu comme l'organisme de référence en matière de radioprotection pour les RNI dans les années 1980. Elle a poursuivi cette activité jusqu'en 1993 dans le cadre de l'INIRC devenu l'ICNIRP en 1992.

Du côté français, lorsque le Conseil d'administration de la SFRP décide la création d'un groupe de travail sur les RNI suite au succès du Congrès de Nainville-les-Roches en 1978, Annette fait bien sûr partie des membres fondateurs avec G. Plurien et B. Servantie, sous la présidence de A.J. Berteaud. Le groupe cesse de fonctionner pendant quelques années mais renaît en 1993 sous forme d'une section à part entière « la section RNI », dont Annette a fait partie dès sa création en tant que membre actif. Elle a notamment été avec Jacques Joussot-Dubien la cheville ouvrière de la première édition de l'ouvrage Les effets biologiques des rayonnements non ionisants, dont la section prépare aujourd'hui la $3^{\mathrm{e}}$ édition.

La science de la radioprotection dans le domaine des RNI doit beaucoup à Annette Duchêne, mais nous aimerions rappeler aussi, outre ses grandes qualités professionnelles, sa gentillesse, sa sensibilité, et son attention aux détails. C'était un plaisir de discuter avec elle en marge des réunions de la section.

Elle manquera à notre discipline et à tous ceux qui l'ont connue, mais restera, à n'en pas douter, un exemple à suivre pour les nouvelles générations.

La section RNI.

Remerciements à Mike Repacholi, Louis Court, et au club Histoire pour leur contribution.

\section{Georges Marblé}

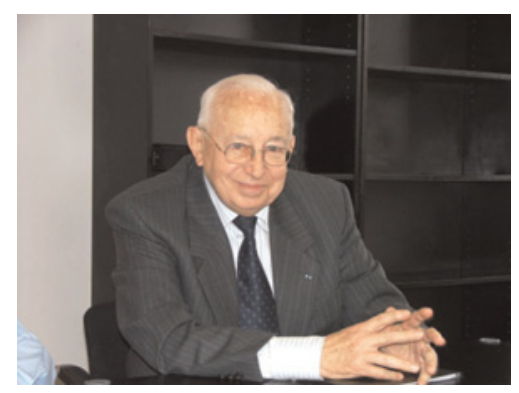

Nous avons appris le décès du professeur Georges Marblé, survenu le 5 janvier 2017 à Aubagne. Il était pharmacienchimiste général des armées, et fut successivement chef de laboratoire au CEA de Saclay et au Centre d'études et de recherches de techniques sous-marines (CERTSM). Il fut président de la SFRP de 1972 à 1973. 